\title{
Artistic Gymnastics as a Semiotic Construct: The Case of the Chinese National Team at the Olympic Games
}

\section{Jiya Wang}

University of Qiqihar

Dawei Yao ( $\nabla$ yaodawei63@yahoo.com )

University of Qiqihar

\section{Research Article}

Keywords: China sports policy, communication, gymnastics, Olympic sports, semiotics

Posted Date: January 27th, 2022

DOI: https://doi.org/10.21203/rs.3.rs-1284585/v1

License: (c) (1) This work is licensed under a Creative Commons Attribution 4.0 International License.

Read Full License 


\section{Abstract}

China is one of the most dynamically developing countries that experienced rapid and tumultuous modernization in the second half of the XX century. This modernization consisted of the sports organization improvement and appropriate target-setting in Olympic sports. Sport is the most important sphere of international communication, and gymnastics pertains to the expression of semiotic units by the movement itself. The purpose of this research is to study a semiotic construct embracing the victories, behavior patterns, and organization of the Chinese gymnastics team at the 2008 Olympic Games in Beijing, where China demonstrated its highest achievements in gymnastics. Another reason for selecting these Olympic Games is that semiotically significant elements of the athlete behavior differ from other contexts and Olympics. Consequently, the latter feature facilitates the segregation of these elements through phenomenological research.

\section{Introduction}

Even during the times of Yuri Lotman, a famous Russian semiotician and the father of semiotics in Russia, semiotics regarded speech and behavior in all its complexity as two separate signs [1]. The semiotics of behavior and various types of its implementation in the cultural sphere is under review along with the semiotics of theater, cinema, painting, architecture, etc. [2]. Semiotic constructs constitute the created forms of content representation. Regarded as "definite texts", they aim to convey certain content to the observer. This content can reflect a semiotic construct both consciously and unconsciously. It may contain partially intentional (established under the will of the person who embodied the construct) and unintentional components, which create an integrity that retains its unique semantic features to the full extent only in a specific context $[3,4]$. These semiotic constructs tend to phenomenologically restore themselves beyond the given temporal, historical, and other contexts. At the same time, they will receive additional modes of understanding due to this new context in which they were restored [5].

There is a close link between nonverbal communication and the semiotics of gesture, sign, and behavior. Yuri Lotman and Boris Uspensky, investigating the semiotic component of behavior among the Russian aristocracy in the 19th century, indicated that a gesture (i.e., a form of giving meaning to a particular type of behavior in a broader context) served to give importance to the behavior within an overall context determined by the individual culture, state, and views [6]. Nonverbal communication constitutes a significant part of behavioral semiotics reflected through several types of communication, including kinesics, proxemics, and takesics [7, 8]. Kinesics studies the system of gestures, poses, body movements, and more, including elements that we use to present ourselves, such as clothing, jewelry, hairstyle, makeup, and related behavioral patterns. These elements of display exist within a single space of human comprehension and have particular meanings. Consequently, the conscious or unconscious choice of clothing, jewelry (and the way a person wears it) is a way of self-presentation, a symbol indicating one's position in society or group, as well as one's values, ideas, etc. [9]. Proxemics refers to the physical interposition of communication objects in space, considering physical space and distance as a form of significance. For example, people who stand very close to each other are more likely to be in a closer, 
possibly friendly, or intimate relationship. Society and habits determine the distinctive features of spatial behavior: who and under what circumstances can put a hand on someone's shoulder, hug another, and how tightly. What is natural for a sibling a close friend may not have the right to do [10]. Takesics studies touch and mutual exchange of various kinds as a form of communication and a specific symbolic sphere. For instance, having a special someone in close proximity can facilitate the course of the disease, invoke specific physiological sensations, etc. As the researchers admit, this area has a significant practical impact but remains insufficiently studied [9].

The rapid economic growth of the PRC in the 1980s and its opening-up policy went in line with a unified national policy. As Chinese researchers affirm, the creation of a certain reputation and changing the image of China and representatives of Southeast Asian countries in world culture, sports, and collective consciousness was the most important part of that policy $[11,12]$. The Chinese government deliberately sought to destroy the myth of a "weak Oriental man" and demonstrate a strong, beautiful, and healthy nation to the whole world [12]. The gradual improvement of nutrition, health maintenance, and social wealth in the regions with advanced development has given the Chinese government a substantial basis for demonstrating the capabilities of the developing Chinese society [11]. In 1979, China resumed membership in the International Olympic Committee and participated in the 1984 Summer Olympics in Los Angeles for the first time. The PRC established the General Administration of Sport (GAS), the purpose of which was to promote China to one of the highest places in terms of the number of medals won [13]. The country has made many significant decisions on the resource allocation for this task. In particular, China prioritized the Summer Olympic Games preparation and focused on those sports where small investments were possible with a quick return in the form of victories. Many modern researchers in sports history criticized this investment approach [14-16].

The concept of "Zhuguo Tizhi" emerged after the 2000 Sydney Olympics. This concept required the local and central authorities to effectively direct (usually limited) financial, human, and scientific resources to support elite sports and achieve success at the Olympic Games, along with the "glory of the nation" [12]. The context of the situation and the use of terms, such as "success" and "fame," indicate the exclusively semiotic nature of the said concept. The task was to create a certain semiotic status, as winning medals at the Olympic Games does not bring any practical benefits for the Chinese economy, does not create new technologies for further implementation in other spheres, and has an exclusively iconic meaning.

Whereas these terms can be economically measured, they have only an illustrative connotation and relate to an abstract symbolic success $[8,17]$.

Moreover, many researchers emphasize the significance of state funding regarding the Olympic Games. Thus, the development of elite sports and coaching in China predominated over other spheres. The Olympic Games is the only game that unites all nations and simultaneously includes competitions in almost all existing sports $[13,18]$. At the same time, the most significant rating for the Olympics is the overall country ranking and not the place of an individual team or athlete. It is due to the fact that all teams are national: they do not relate to a club or local community, so each athlete "defends the honor of the country" in every individual competition. Therefore, the Olympic Games is the only sports competition 
conducted between countries, rather than teams or athletes, and their symbolic and semiotic context is the most significant among others [17]. That is why it is important to analyze the semiotic construct within the representation of a national sports team in an international competition.

According to Chinese sports researchers, artistic and rhythmic gymnastics were among the few sports that received the most significant funding and preferential support in the hope of achieving the maximum number of awards. Consequently, gymnastics remarkably resulted during the Olympic Games in Beijing 2008 , implementing the task set for the Chinese sports $[19,20]$.

\subsection{Literature overview}

The analysis of the remarkable Chinese athletic performance after the country's return to the International Olympic Committee arouses the interest of researchers from many fields. In particular, they apply the most prevalent econometric model, an extended version of the Tobit model developed primarily for winter sports [21, 22]. This model evaluates and tries to predict the future sports achievements of certain countries within the Olympic sports through the analysis of econometric data, such as the size of GDP per capita, the number of population and sports participants, the availability of sports resorts, and other factors [21]. The PRC has emphasized the significance of summer sports since the very beginning of the Olympic sports development at the domestic level; hence this sphere continues developing. Thus, the transfer of this model to summer sports seems quite adequate. Nonetheless, the prediction of the results of the Sochi Olympics proposed by the authors of this research turned out to be far from reality.

Many researchers firmly associate the semiotic concepts of China's modernization with its sports achievements $[12,19,20,21]$. The implication of Chinese culture in the international context and the peculiarities of its history contributed to China's involvement in the modern Olympic movement [19]. China strives to symbolically convey its overall modernization to the world community, same as for its citizens. Therefore, sport acts as a means of the most important political and ideological communication. As previously in the case of the Soviet Union, sports achievements are a symbolic representation of modern China, i.e., a pure semiotic construct [23].

The researchers point out that the Chinese sports organization aspired to achieve mainly non-sporting goals: representing the country and increasing its fame. This task requires more winnings, more awards, and flawless performance in a relatively short time, along with a constant increase in the number of wins compared to other countries [21]. Since, according to game theory, Olympic competitions are a game with one winner, it is a displacement task. Therefore, the country has to educate strong elite athletes to achieve this task. At the same time, the state sports system should work in a rigorous selection mode of optimal representatives who can potentially achieve the goal set by the government [24, 12]. Such a sports policy may lead to unexpected results. Focusing on elite athletes, who make up a relatively limited number, reduces the chances for more willing people to get into professional sports. On the other hand, a country striving for the highest achievements needs to create the widest possible range of people who play sports seriously and regularly, i.e., generate a sufficient number of future champions [20,25]. In turn, such a policy forms a number of semiotic constructions within the framework of internal ideology and mass 
consciousness. Consequently, it can reflect in the consciousness of athletes and partially enter into the semiotic constructions demonstrated for the external observer. A good example is the aestheticization of sports, including the favoritism of multiple winners as cult figures (they represent the country rather than themselves) in society $[18,20]$.

Many semioticians consider gymnastics a special semiotic form of sport that facilitates demonstrating an implied meaning through body movement, for example, through dancing [26, 27]. The great actors of the silent film era, who did not yet have other means of communication, communicated with their viewers in a similar fashion [8]. However, the analysis of the content of rhythmic gymnastics performances at the Olympic Games, in this case, plays a secondary role, whereas the athletes themselves, the structure of their victories at the Games, their behavior, and demonstrated public gestures more fully and directly represent the intended content $[28,29]$.

The organization of the National Games of China (NGC) is a very significant element in the semiotic representation of sports in the country. The NCG (an analog of the Olympic Games) operates within the country and covers a huge number of participants. According to researchers and national statistics of the People's Republic of China, the National Games regularly involves about 10 thousand athletes, more than 30 sports disciplines, and more than 350 types of competitions [15]. That is why these games semiotically reflect the Olympic Games at the domestic level as a form of preparation of athletes for international competitions. The PRC strives to take a dominant position in any field, including sports. China takes an active part in organizing and promoting the Asian Games, which semiotically reflect the Olympic Games at the regional level [14, 30].

The purpose of this study is to conduct the phenomenological and semiotic analysis of behavior patterns and public representation strategies executed by sports competitions, which demonstrate the meanings embedded in the politics of a particular country or nation. Nowadays, there is a significant gap in academic research regarding the semiotic studies of sports and sports as a form of ideology and politics on an international scale. Consequently, this study aims to fill this gap.

\section{Method And Materials}

This research includes the semiotic analysis of behavior artifacts, such as photos, videos, and other materials representing the behavior, performance, and sport achievements of Chinese gymnasts at the Beijing 2008 Olympics. Phenomenological reduction is the underlying method of this research. It stipulates that the semiotic basis is extracted from the observed facts and the diverse forms of behavior are reduced to a semiotic construct. According to the study hypothesis, this semiotic construct may be the cornerstone of this behavior.

This phenomenological hypothesis builds on the aforementioned studies, which directly indicate the presence of an intensional semiotic structure, including certain symbolic messages and conscious goals conveyed by Chinese gymnasts at the Olympic Games. The political authorities of the People's Republic 
of China, the Government and the General Administration of Sport of the People's Republic of China formulated this message, which subsequently manifested itself in the process of sports competitions.

Moreover, the present research provides a comparative analysis. It builds on data from Chinese and Western field studies and historical research in semiotics, the history of sports, and sports policy of China.

The present research also conducts additional verification of intuitive semiotic observations through the statistical study of photographic materials from the Beijing 2008 and Rio de Janeiro 2016 Olympic Games. The reason behind selecting these two competitions is the contrasting performance of Chinese athletes in these Olympics. In 2008, the Chinese gymnasts won 11 out of 18 gold medals, while the 2016 Rio competition was less successful. The gap was significant. The Chinese gymnastics team brought home zero gold medals and just one bronze one, earned by Li Dan in the women's trampoline competition.

As can be seen, the selected samples of Olympics are the semantic "poles", one pole is the Beijing competition with the maximum achievements and the other is the Rio de Janeiro competition with the minimum achievements. Hence, the null hypothesis was formulated, which holds that there are no statistically significant differences in the percentage ratio between the number of photos depicting semiotically significant behaviors of Chinese gymnasts at both Olympics. In other words, the difference in the number of informative photos between the two groups is random. This hypothesis was tested by applying a modified Shapiro-Wilk test. For this, the following encoding was implemented: the value of 1 accounts for the presence of the desired pattern, and the value of 0 corresponds to its absence.

The photographs used in the study depict athletes in semiotically significant contexts: the awarding ceremony, events in the arena, and their interaction with the coach, teammates and rivals. This approach has its limitations. The major one is the limited sample size: the study does not cover all video and photo materials out there. However, it is safe to extrapolate visual evidence from the world news agencies to athlete behavior in semiotically significant contexts because of behavioral consistency they tend to demonstrate in those situations.

Semiotically significant behavior does not necessarily have to cover the entire time spent in this context. Moreover, executing a behavior other than the expected semiotically significant one may indicate an accentuated semantic value of semiotic behavior. In other words, a person who has to behave in a certain way will relax at some point and accidentally demonstrate a type of behavior, which is completely different from the one exhibited previously. It is important to keep in mind that semiotic studies are not identical to psychological or semantic ones. Thus, it may be difficult to deploy statistical tools, which brings us to another limitation.

The sample includes photographs depicting 24 members of two Chinese gymnastics teams (12 athletes per team), both male and female, who reached All-Around Finals in 2008 and 2016 competitions. In total, there were 386 photos from Beijing and Rio de Janeiro (32 - 33 photos per athlete). These photos were 
randomly selected from the total sample of visual materials available to the researcher. The final sample accounts for about $40 \%$ of the total sample, which makes it statistically significant.

\section{Results}

In relation to Chinese athletes in the award-winning scenario (2008 Beijing Olympics), there were several recurring patterns of behavior observed in both sexes within the same contexts. Similar patterns were seen during the Athens Olympics in 2004, where the Chinese national team also achieved impressive results [11]. For comparison, photos from Rio showed substantial differences in behavior and basic gestures of Chinese athletes, who realized that no awards have been earned.

Consequently, the described semiotic construct with its components and features is a recurring phenomenon associated with unconditional victory and significant advantage over rivals. In this study, these types of behavior are semiotically underpinned, which means that a focus lies on a meaningful content designed to convey a certain meaning to the observer, rather than its psychological foundation. Previously observed and analyzed data will help to reconstruct this meaning.

Semiotically significant gestures and signs expressed by the Chinese gymnasts differ from those demonstrated by the representatives of other countries. Moreover, we can observe a typical semiotic form of behavior both among Chinese athletes and gymnasts of other countries. At the same time, the patterns are similar among those countries but significantly different from the Chinese ones.

At the same time, Chinese athletes look either slightly down or forward, but rarely up. Such a physiological status cannot be interpreted as a result of a specific sports training because other gymnasts with a similar and higher level of training behave differently. Other semiotically significant public gestures also endorse this type of behavior. Meantime, athletes in a semiotically insignificant context are more relaxed and can exert "mistakes" in behavior management, i.e., go beyond the semiotic bodily context intended for public presentation.

Thus, Chinese athletes often kiss their medals and demonstrate them at the head level without changing the position of the award, as if they were symbolically identifying it with their faces. The semiotic nature of this gesture is quite distinctive amid the behavior of representatives of other countries, who can raise their awards high, touch them, shift them, show them to each other and even wave them, which is unlikely for the representatives of China. Chinese gymnasts also raise only one hand, usually the right one, to greet the audience or celebrate their victory. Furthermore, Chinese athletes maintain a straight posture and keep their face orientation unchanged; they tend to show minimal movement and even stand still.

The set of public gestures demonstrated by Chinese athletes during the Beijing 2008 Olympic Games is sharply different from those seen in other Olympics, in particular, in Rio de Janeiro, where the Chinese team received poor results. This collection of gestures coincides with the basic patterns highlighted in previous surveys conducted among the Chinese psychologists, who served the Olympic team. Considering the most important collective interpretations, there is a strong emphasis on the need to pay 
tribute to the administrative authorities and coaches while prioritizing teamwork. In fact, it is possible to directly trace the semiotic representation of all the described patterns in the public gestures of athletes.

The control of facial expressions presupposes the absence of exuberant and sincere joy in the event of victory. Such a demonstration of happiness would humiliate opponents and those close to them. Similarly, in case of one's own defeat, one should remain indifferent and stoic without demonstrating displeasure or confusion. The primacy of collectivism excludes the demonstration of personality traits in behavior. In other words, it excludes the demonstration of behavioral patterns that could be read by others differently than in the context of the meaning that the whole group (that a person belongs to) should convey. In this case, a group of gymnasts had to reach the "glory of the nation" and prove how strong China is (hence the persistent symbolic identification of medals with their faces). It is a medal that is important here as the conquest of the country rather than the athlete's personality. This approach sharply contrasts with the Western, European, and American paradigms. The latter entails highly individualized forms of athletes' behavior in most contexts because it is personality, uniqueness, and identity that stand out as the source of the overall strength of Western civilization.

Unlike their colleagues from other countries, Chinese gymnasts never hug closely. They demonstrate a clear separation and distance from their rivals at the level of proxemics. A good example in the context of awarding is their behavior on the pedestal. This is precisely a semiotic and not exclusively a psychological or political phenomenon. In this case, the political and psychological phenomena carry a semiotic context. An athlete, as an individual, can feel united and demonstrate communion with another athlete belonging to the same corporation of elite sportsmen. However, in this case, the Chinese delegation had to convey the meaning of the Chinese victory. They had to prove the advantages of the Chinese and the alignment of their semiotic status in comparison with other nations not only within the sports context but in the context of the world competition for the country's status at the global level. Therefore, distancing as a means of the aforementioned demonstration is inevitable at the physical level. The Chinese gymnasts do not experience any negative emotions towards other athletes and behave sincerely and friendly. Such a behavior further strengthens this suggestion. At the same time, being a part of a broad symbolism, they act as translators of a certain semiotic construct and perform a specific function assigned to them within this construct.

As a semiotic fact, clothing has been one of the most important means of communication throughout all the ages [2]. The Chinese uniform at the Beijing Olympics has the colors of red and gold, which is a frank and demonstrative symbol. The red color symbolizes the dominant communist ideology of China. Moreover, it is the most powerful traditional Chinese benevolent symbol. In the traditional semiotic system of China and the Far East, red is the color of health, strength, activity, longevity, and power [31]. It is an ideal representation for symbolizing sports, its achievements, and the idea of modern China's renewal and the country's accomplishment for greater power. The gold color represents the color of victory. In other words, it is the color of winning gold medals as the main goal set for the Chinese delegation at these Games. 
Gymnastics, as a sports discipline, and its representatives at the Olympic Games also act as a whole message at the level of the overall achievements of the team. The awards distribution additionally confirms this suggestion $[32,20]$. Thus, the individual competitions brought most of the medals, while the team performances of Chinese gymnasts (in particular, in rhythmic gymnastics) turned out to be very weak. The Chinese gymnast was even included in the list of the best in the individual rhythmic gymnastics competition to maintain the honor of the host country of the Games [12]. The researchers pointed out that this result reflects the specific organization of Olympic and professional sports in the PRC, where team sports were practically excluded from state sports financing and management and moved into the sphere of commercialization. Whereas football, as the most popular game that attracts significant resources around the world, began to rapidly develop, then less popular rhythmic gymnastics remained isolated and significantly lagged in the training of athletes [12, 24]. Nevertheless, all the elements of the general semiotic construct described above are common for both the representatives of the losing Chinese team and winning individuals in gymnastics.

Based on the Shapiro-Wilk test results, both samples, images from the Beijing Olympics $(W=.122)$ and images from the Rio Olympics ( $W=.189)$, are normally distributed $(p \leq .05)$. Hence, they can be used in the Student's t-test. The results from the semiotically significant behavior assessment are presented in Table 1.

Table 1

The presence of semiotically significant patterns of behavior among gymnasts in photos from the Olympics in Beijing and Rio de Janeiro.

\begin{tabular}{|llll|}
\hline & & Beijing & Rio-de-Janeiro \\
\hline contains a pattern & photo, $\%$ & $98,25 \%$ & $17,75 \%$ \\
\cline { 2 - 4 } & participants, $\%$ & $100 \%$ & $33,33 \%$ \\
\hline
\end{tabular}

As shown in Table 1, athletes on Beijing photos demonstrate specific semiotic patterns in almost all cases, whereas athletes in Rio who exerted these behaviors account for just $33.33 \%$ of the total athletes, or $17.75 \%$ of the sample photos. Accordingly, the high percentage of semiotic patterns displayed during the Beijing 2008 Olympic Games could be linked to the high performance of Chinese gymnasts. However, the nature of this connection is not revealed statistically. Therefore, the hermeneutical and phenomenological analysis enables the aforementioned study of its assumptions. Accordingly, Table 2 shows the results of the Student's t-test for the null hypothesis verification.

Table 2

Student's t-test results

for the null hypothesis

testing.

\begin{tabular}{|lll|}
\hline$d t$ & $\boldsymbol{t}$ & $\boldsymbol{p}$ \\
\hline 385 & 1.966 & .041 \\
\hline
\end{tabular}

Page 9/14 
The studied patterns displayed in the photographic samples from the two Olympic Games show statistically significant differences. Consequently, the null hypothesis should be discarded.

\section{Discussion}

It is worth mentioning that for modern China, the ideological component of many spheres, especially those related to foreign policy, is more significant in comparison with the developed Western countries or Japan or South Korea $[11,30]$. Nevertheless, there is a certain semiotic construct of greater or lesser clarity and depth formed during the presentation of sports achievements of any country at the Olympic Games $[17,23,33]$. In the case of China, this construct has the properties of high structuring, presence of rigid connections between individual elements, and even some defiant evidence, which is one of its most significant semantic features. Chinese researchers of the history of sports emphasize the significance of sports achievements and confirm the presence of a clearly expressed intention of the Chinese political and sports authorities to convey to the world community their ideas $[13,15,16]$.

The previous study on socio-cultural factors that influenced the delivery of services to Chinese athletes by sport psychologists indicates the following facts. Those psychologists who were not able to convey and form the necessary patterns (that ensure the communication of the semiotic construct) in their wards' behavior received a warning; and the administration corrected their activity in the required direction [25]. In this case, this research concerns the formation of the semiotic background of athlete's behavior (which was conscious and possibly prescribed by the administration), rather than the evaluation methods of athlete training.

Many researchers infer that the focus of the Chinese support system of sports on winning as many medals as possible was an erroneous choice $[14,11,19]$. This requirement, accordingly, caused an early understanding of the sports administration regarding the need to develop elite sports and the coaching component. It further resulted in the displacement of many sports almost exclusively into a commercial form of existence and led to the lack of incentives for young talented athletes and the lag of amateur sports. Moreover, it encouraged manipulation and corruption in sports management $[13,16]$.

The researchers also point out that the formed model of sports development and the semiotic model conducted by the PRC comes to a natural self-denial. The Chinese type of sports management leads to the formation of a cohort of increasingly wealthy and mobile individualists, high-class elite athletes, and their coaches who are no longer interested in demonstrating the described semiotic model and can no longer be a part of it [12]. It is exactly their denial and the changes described above that also indirectly confirm the possibility of identifying the semiotic construct within this study.

\section{Conclusion}

The dynamic development of China in the XX century resulted in a specific sports policy, which, in turn, generated a unique set of signs used by athletes for self-presentation on the international arena. The 
country has set ambitious goals to form a new image of the country and its representatives and win as many medals as possible through participation in the Olympic Games. Regarding the fact that sport is the most important sphere of international communication, gymnastics pertains to one of the best examples of the semiotic expression through its performance. Consequently, using phenomenological reduction, we have defined the key features of the semiotic construct, which translates through the sports victories, individual and collective behavior, and the organization of the Chinese gymnastics team at the Olympic Games in Beijing 2008. The results of China at the Beijing Olympics reaffirm its significance as an example of the successful implementation of the country's goals. It is worth mentioning that semiotically significant elements of athlete behavior differ from similar behavior in other contexts during other Olympics. Thus, it is possible to distinguish those components through phenomenological research. The semiotic cores of collectivism, the control of facial expressions in victory and defeat, the demonstration of respect for the sports administration authorities and coaches, rigidly sequenced and consistent interpersonal communication and self-control are at the heart of the studied construct. This research also provides the analysis of its external manifestations, such as public gestures, proxemics, clothing and color choices.

\section{Declarations}

\section{Author contributions}

JW: Conceptualization, Methodology, Data curation, Writing- Original draft preparation, Investigation. DY: Supervision, Writing- Reviewing and Editing.

\section{Funding}

General Undergraduate Colleges and Universities Youth Innovative Talent Training Program Project in Heilongjiang Province in 2020, China (Grant No. UNPYSCT-2020079).

Provincial undergraduate university basic research business fee Youth Innovative Talent Project in Heilongjiang Province in 2021, China (Grant No. 135509279).

Educational Science Research Project of Qiqihar University in 2020 (Grant No. GJSKZX202001).

General project of College Student Innovation Project in Heilongjiang Sort out and study the symbols of ice and snow sports in Heilongjiang Province (Grant No. 202110232051).

\section{Conflict of interests}

This research has no conflict of interests. 


\section{Data Availability}

Data will be available on request.

\section{References}

1. Tamm, M. (2019). Juri Lotman-Culture, Memory and History: Essays in Cultural Semiotics. Springer Nature.

2. Hervey, S. (2016). Semiotic perspectives. Routledge.

3. Marrone, G., \& Mangano, D. (2015). Brand language. Methods and models of semiotic analysis. In G. Rossolatos, Handbook of Brand Semiotics (pp. 46-88). Kassel University Press.

4. Picione, R. D. L., Martino, M. L., \& Troisi, G. (2019). The semiotic construction of the sense of agency. The modal articulation in narrative processes. Integrative Psychological and Behavioral Science, 53(3), 431-449.

5. Keane, W. (2018). On semiotic ideology. Signs and Society, 6(1), 64-87.

6. Monticelli, D. (2016). Critique of ideology or/and analysis of culture? Barthes and Lotman on

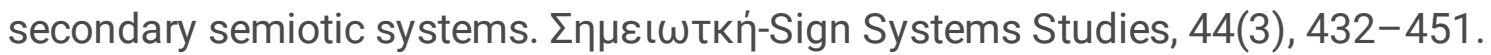

7. Spapé, M., Harjunen, V., Ahmed, I., Jacucci, G., \& Ravaja, N. (2019). The semiotics of the message and the messenger: How nonverbal communication affects fairness perception. Cognitive, Affective, \& Behavioral Neuroscience, 19(5), 1259-1272. https://doi.org/10.3758/s13415-019-00738-8

8. Sheets-Johnstone, M. (2019). The silence of movement: A beginning empirical-phenomenological exposition of the powers of a corporeal semiotics. The American Journal of Semiotics. https://doi.org/10.5840/ajs20196550

9. Eco, U. (1979). A theory of semiotics (Vol. 217). Indiana University Press.

10. Wee, L., \& Goh, R. B. (2019). Language, space and cultural play: Theorising affect in the semiotic landscape. Cambridge University Press.

11. Ren, T. (2019). A special salience: media, iconography, nationalism: modern Chinese Olympic Games and heroes as soft power 'projectiles'. In Media, sport, nationalism: East Asia: soft power projection via the modern Olympic Games (pp. 49-82). Berlin: Logos Verlag.

12. Ma, Y. (2020). Governance of Olympic Elite Sport and Professional Football in China: Studies on the National Games of China and the Chinese Super League (Doctoral dissertation). https://doi.org/10.15495/EPub_UBT_00004931

13. Zheng, J., Lau, P. W. C., Chen, S., Dickson, G., De Bosscher, V., \& Peng, Q. (2019). Interorganisational conflict between national and provincial sport organisations within China's elite sport system: Perspectives from national organisations. Sport management review, 22(5), 667-681. https://doi.org/10.1016/j.smr.2018.10.002

14. Zheng, J., Tan, T. C., \& Bairner, A. (2019). Responding to globalisation: The case of elite artistic gymnastics in China. International review for the sociology of sport, 54(5), 536-556. 
https://doi.org/10.1177\%2F1012690217730679

15. Ma, Y., \& Kurscheidt, M. (2019). The National Games of China as a governance instrument in Chinese elite sport: an institutional and agency analysis. International Journal of Sport Policy and Politics, 11(4), 679-699. https://doi.org/10.1080/19406940.2019.1633383

16. He, J., Montez de Oca, J., \& Zhang, L. (2020). Why Team China suffered its worst performance in artistic gymnastics at Rio 2016? International Journal of Sports Science \& Coaching, 15(5-6), 696705. https://doi.org/10.1177\%2F1747954120939347

17. Lee, J. W. (2017). Semiotics and Sport Communication Research: Theoretical and Methodological Considerations. Communication \& Sport, 5(3), 374-395.

https://doi.org/10.1177\%2F2167479515610764

18. Tan, T. C., \& Houlihan, B. (2013). Chinese Olympic sport policy: Managing the impact of globalisation. International review for the sociology of sport, 48(2), 131-152.

https://doi.org/10.1177\%2F1012690212445169

19. Jiang, Y. (2018). National renaissance, International assertion, new global image: China's involvement in the modern Olympic games: media projection. In Media, Sport, Nationalism: East Asia: Soft Power Projection via the Modern Olympic Games (pp. 223-258). Logos Verlag Berlin $\mathrm{GmbH}$.

20. Shen, L. (2020). Olympic Strategy, Nationalism and Legitimacy: The Role of Ideology in the Development of Chinese Elite Sports Policy in the First Reform Decade, 1978-1988. The International Journal of the History of Sport, 37(sup1), 26-40. https://doi.org/10.1080/09523367.2020.1737021

21. Andreff, W. (2013). Economic development as major determinant of Olympic medal wins: predicting performances of Russian and Chinese teams at Sochi Games. International Journal of Economic Policy in Emerging Economies, 6(4), 314-340.

22. Amore, M. D., \& Murtinu, S. (2021). Tobit models in strategy research: Critical issues and applications. Global Strategy Journal, 11(3), 331-355. https://doi.org/10.1002/gsj.1363

23. Hong, F., Wu, P., \& Xiong, H. (2005). Beijing ambitions: An analysis of the Chinese elite sports system and its Olympic strategy for the 2008 Olympic Games. The international journal of the history of sport, 22(4), 510-529. https://doi.org/10.1080/09523360500126336

24. Junio, E. L., \& Rodrigues, C. (2017). The Chinese football development plan: Soft power and national identity. Holos, 5, 114-124. https://doi.org/10.15628/holos.2017.5750

25. Si, G., Duan, Y., Li, H. Y., Zhang, C. Q., \& Su, N. (2015). The influence of the Chinese sport system and Chinese cultural characteristics on Olympic sport psychology services. Psychology of Sport and Exercise, 17, 56-67. https://doi.org/10.1016/j.psychsport.2014.08.008

26. Peterson, J. V., Thornburg, A. M., Kissel, M., Ball, C., \& Fuentes, A. (2018). Semiotic mechanisms underlying niche construction. Biosemiotics, 11(2), 181-198. https://doi.org/10.1007/s12304-0189323-1 
27. De Luca Picione, R. (2021). Models of semiotic borders in psychology and their implications: From rigidity of separation to topological dynamics of connectivity. Theory \& Psychology, 31(5), 729-745. https://doi.org/10.1177\%2F0959354320947184

28. Peuronen, S. (2017). Heteroglossic and multimodal resources in use: participation across spaces of identification in a Christian lifestyle sports community. In Jyväskylä studies in humanities. University of Jyväskylä.

29. Dovchin, S., Pennycook, A., \& Sultana, S. (2018). Transglossia and Sports: Men Talk and Masculinity. In Popular Culture, Voice and Linguistic Diversity (pp. 111-137). Palgrave Macmillan, Cham.

30. Chiu, H. J., Lee, P. C., \& Jiang, R. S. (2021). Should it be "Chinese Taipei” or "Taiwan"? A strategic relations analysis of name rectification referendum for the 2020 Tokyo Olympic Games. Sport in Society, 24(12), 2159-2183. https://doi.org/10.1080/17430437.2021.1991323

31. Okafor, M. U. (2020). Chinese colour symbolism: It's significance to the igbo. Odezuruigbo Journal, $4(1), 215-225$.

32. Kerry, V. J. (2017). The construction of hegemonic masculinity in the Semiotic Landscape of a CrossFit 'Cave'. Visual Communication, 16(2), 209-237. https://doi.org/10.1177\%2F1470357216684081

33. Bowman, P. (2020). From chop suey to chop-socky: the construction of Chineseness in British television adverts. JOMEC Journal, 15, 1-29. http://dx.doi.org/10.18573/jomec.19 